\title{
Les monuments romains
}

Martial Monteil

\section{OpenEdition}

Journals

Édition électronique

URL : http://journals.openedition.org/rao/3190

DOI : 10.4000/rao.3190

ISBN : 978-2-7535-5014-8

ISSN : 1775-3732

Éditeur

Presses universitaires de Rennes

Édition imprimée

Date de publication : 31 décembre 2015

ISBN : 978-2-7535-5012-4

ISSN : 0767-709X

Référence électronique

Martial Monteil, "Les monuments romains », Revue archéologique de l'Ouest [En ligne], 32 | 2015, mis en ligne le 28 avril 2016, consulté le 02 mars 2021. URL : http://journals.openedition.org/rao/3190 ; DOI : https://doi.org/10.4000/rao.3190

@ Presses universitaires de Rennes 
des constructions pouvant, pour certaines, matérialiser des lieux de pouvoir. Là encore des exemples simples viennent illustrer les propos des auteures.

Le lecteur est ensuite conduit en ville où toujours selon un schéma chronologique, on tente de lui montrer les développements urbains du premier Moyen Âge où la ville est principalement qualifiée de " chrétienne » jusqu’au second Moyen Âge où s'invente la ville moderne. Tout comme pour le chapitre précédent, les activités économiques comme les lieux d'habitat ou de travail sont décrits simplement.

À présent que le lecteur est plus familiarisé avec la période, les auteures attirent cette fois-ci son attention sur l'ingéniosité dont il est fait preuve au Moyen Âge dans un chapitre carrément intitulé " une première révolution industrielle »! De manière dynamique, un certain nombre d'innovations médiévales sont déclinées dans les domaines aussi variées que l'agriculture, les artisanats des matières animales, les arts du feu, l'exploitation des matières premières et leur transformation. Ce chapitre, à la suite du premier, attire l'attention sur la gestion de l'environnement et l'épuisement aussi bien des sols, que des espèces végétales et animales.

Mais qui sont donc ces femmes et ces hommes du Moyen Âge? C'est dans un chapitre consacré à " une société multiculturelle et métissée " que les auteurs présentent aussi bien les pratiques funéraires et ce qu'elles enseignent sur les sociétés, le niveau de santé des populations en évoquant notamment le handicap et son traitement au Moyen Âge à l'aide de quelques exemples archéologiques, que la diversité d'origine des populations médiévales métropolitaines et leur intégration.

Pour achever ce parcours initiatique du Moyen Âge, les auteurs invitent allègrement le lecteur à bouger en empruntant un réseau viaire beaucoup plus important que le laisse entendre la tradition, en montant sur des embarcations qui naviguent aussi bien sur les cours d'eau, les littoraux ou en haute mer, en s'arrêtant dans des ports, en rejoignant des foires, en suivant les moines, les pèlerins ou les marchands.

Lorsque le lecteur ferme le livre, il a du mal à quitter le dynamisme médiéval qui lui a été donné de voir! Le pari des auteures de présenter un autre Moyen Âge est à coup sur gagné. L’obscurité dont était affublée cette période de l'histoire disparaît au profit d'une lumière, un peu trop brillante peut-être, qui montre une société adaptable, inventive, intégratrice. De l'aveu même des auteures, certains aspects ont volontairement été délaissés. Si la collection ne permet en effet pas de tout traiter, l'évocation de certains aspects de la société médiévale (guerre, église) auraient probablement permis de contrebalancer ce tableau du Moyen Âge qui peut parfois apparaitre du coup un peu surexposé. Soulignons avec force la qualité de la présentation didactique des résultats les plus récents de la recherche avec de réelles originalités. L'ensemble du propos est appuyé, toujours fort à propos, par une illustration de choix et de grande qualité qui contribuent grandement à celle de l'ouvrage. Il est par ailleurs plaisant d'y trouver un certain renouvellement par rapport aux ouvrages du même éditeur.

Les professionnels trouveront par-ci, par-là des approximations, des développements un peu vagues notamment sur la ville du premier Moyen Âge, qu'importe. Il se trouve là un ouvrage, pas trop cher, qui permet à tous de pouvoir se faire une idée relativement précise, à l'aide des résultats les plus récents de la recherche archéologique, d'une longue période, loin des sempiternels clichés qui malheureusement sont toujours à l'honneur des animations culturelles estivales et des parcs d'attractions. On ne peut, ici, que saluer les auteures qui ont toujours réussi à exposer et démontrer les faits, sans concession, pour le savoir du plus grand nombre.

Édith Peytremann (Inrap, UMR 6273)

Tendron G., 2014 - Yzeures-sur-Creuse (37). Les monuments romains, Chauvigny, Association des Publications Chauvinoises, coll. «Memoria Momenti; $32 », 95$ p. (ISBN 979-10-90534-24-7).

Dans cet opuscule richement illustré, Graziella Tendron rend compte de l'étude qu'elle a consacrée à des fragments d'architecture romaine découverts à la fin du XIX ${ }^{e}$ siècle, lors d'une réfection de l'église médiévale d'Yzeures-sur-Creuse. Leur découverte attira le révérend père jésuite Camille de la Croix, fondateur de l'archéologie poitevine, qui organisa une campagne de fouille en février 1896 permettant d'extraire entre 85 et 95 blocs. 52 d'entre eux sont conservés au sein du musée Minerve du bourg et ont fait l'objet d'une nouvelle présentation en 2014, donnant l'occasion de les réexaminer.

Ces membra disjecta correspondent en fait, si l'on excepte quelques morceaux de sarcophages du haut Moyen Âge, à des portions de monuments relevant d'un sanctuaire antique qui, après son abandon, ont été récupérés pour être remployés, très vraisemblablement, au sein d'une église mérovingienne antérieure à l'actuelle. 21 blocs permettent 
de restituer un pilier votif dont la hauteur peut être estimée à environ $9 \mathrm{~m}$ et qui avait fait l'objet d'une première étude en 1972. Dédié à Jupiter et supporté par un piédestal, il est constitué de trois niveaux sculptés en bas-relief figurant en alternance des divinités et des scènes mythologiques. S'y ajoutent 3 blocs d'une dédicace à Minerve et aux divinités des empereurs. Cette inscription témoigne du financement de la construction d'un temple par deux frères, citoyens romains, suivant la pratique courante de l'évergésie. Les autres blocs n'avaient pas, jusqu'alors, fait l'objet d'une publication. 24 d'entre eux appartiennent à un temple de forme polygonale, dont l'architrave et une partie des pilastres et de l'entablement peuvent être restituées. 2 autres, enfin, participent du couronnement d'un autel réservé aux sacrifices lors des cérémonies.

L'ensemble fait l'objet d'une analyse minutieuse qui combine texte, dessins et photographies, parmi lesquelles plusieurs clichés inédits issus des archives du père de la Croix. L'enquête est complétée par une approche des techniques de taille et de mise en ouvre de ces blocs en calcaire et se conclut par une comparaison pertinente avec le sanctuaire picton du Gué-de-Sciaux à Antigny (Vienne). Celui d'Yzeures-sur-Creuse, dont les restes trahissent l'ampleur et la qualité des ornamenta, se trouvait quant à lui en limite sud du territoire turon, au sein d'une probable agglomération antique. Les datations stylistiques suggèrent une édification du temple et du pilier votif à la fin du $\mathrm{II}^{\mathrm{e}}$ siècle ou au début du III ${ }^{\mathrm{e}}$ siècle apr. J.-C.

Conçu tout à la fois comme une étude archéologique complète et rigoureuse mais aussi comme un guide proposé aux visiteurs du musée, cet ouvrage au prix modique s'adresse à tous ceux qui s'intéressent à l'architecture religieuse des Gaules romaines, mais aussi à l'histoire de l'archéologie, illustrée ici par la reproduction de nombreuses photographies prises au moment des fouilles de 1896 et par la narration des conditions dans lesquelles celles-ci se déroulèrent.

Martial MonTeIL

(université de Nantes - LARA-UMR 6566 CReAAH)

BaCHELIER Julien, 2014 - Villes et villages de Haute-Bretagne (XI'-début XIV siècles). Analyses morphologiques, supplément AK des Dossiers du Centre Régional d'Archéologie d'Alet, 242 p. (ISSN 0399-6662).

Cette publication est issue des annexes d'une thèse d'histoire médiévale ${ }^{1}$, soutenue en 2013 à Rennes 2 sous la direction de Daniel Pichot, qui en signe la préface. L'auteur, agrégé d'histoire, y présente l'ensemble des plans de villes, bourgs et villages - voire fermes - sur lesquels il a travaillé, en Haute-Bretagne.

Eu égard au nombre de plans analysés et présentés, cet ouvrage est avant tout un atlas qui constitue la base des analyses morphologiques proposées. La partie cartographique représente, pour cette raison, la majeure partie du livre (195 pages sur 242). La précède une "présentation » d'ordre méthodologique et conclusive (mais assez courte : 17 pages) abordant : le travail de constitution des données (p. 11-13), une brève historiographie des recherches en morphologie (p. 14-18), le vocabulaire utilisé dans les fiches établies par localité (p. 19), les difficultés de l'exercice (p. 19-20) et les principaux enseignements que l'auteur tire de ce travail (p. 21-24). Puis suivent les fiches des villes, bourgs et villages de Haute-Bretagne connus dans les sources écrites entre le $\mathrm{XI}^{\mathrm{e}}$ et le début du XIV $^{e}$ siècle. On découvre 173 entrées, par nom de lieux, associant une notice et un plan. Chaque notice adopte

1. «Villes et villages de Haute-Bretagne. Les réseaux de peuplement $\left(\mathrm{XI}^{\mathrm{e}}-\mathrm{XIII}^{\mathrm{e}} \mathrm{s}.\right) »$. une présentation normalisée la situation administrative (département, arrondissement, canton) et ecclésiastique durant les $\mathrm{XI}^{\mathrm{e}}$-XIII ${ }^{\mathrm{e}}$ siècles (diocèse, doyenné), la superficie du site étudié ${ }^{2}$, les analyses morphologiques réalisées par l'auteur et les sources et références bibliographiques à partir desquelles il a travaillé. À ces notices est associé un plan, dessiné sous Adobe Illustrator ${ }^{3}$, à partir du levé du cadastre napoléonien qui représente " la plus ancienne représentation cartographique englobant de manière précise et détaillée toutes les localités étudiées " (p. 13). Si très ponctuellement d'autres documents cartographiques ont été exploités ${ }^{4}$, le cadastre ancien reste la base des analyses morphologiques. L'ouvrage se clôt sur une liste des abréviations, des sources et de la bibliographie.

L'objectif affiché de J. Bachelier est de pallier la sousexploitation du cadastre napoléonien en histoire médiévale qui demeure " peu utilisé » hors des études monographiques et " encore trop souvent dans un seul but illustratif» (p. 9).

2. Mais la méthode utilisée n'est pas précisée alors que l'exercice est délicat pour les agglomérations à la structure éclatée et à l'extension diffuse.

3. On regrettera à ce sujet que ce travail, pourtant commencé au début des années 2000, n'ait pas été réalisé sous SIG afin d'en faire un outil susceptible d'être partagé et enrichi plus facilement.

4. Une copie couleurs sur feuille libre d'un plan de Fougères du $\mathrm{XVIII}^{\mathrm{e}}$ siècle (date non communiquée) est d'ailleurs jointe à la publication. 